\title{
Difference in Binocularity and Ocular Dominance Plasticity between GABAergic and Excitatory Cortical Neurons
}

\author{
Katsuro Kameyama, ${ }^{1,2 \star}$ Kazuhiro Sohya, ${ }^{1 \star}$ Teppei Ebina, ${ }^{1,3}$ Atsuo Fukuda, ${ }^{4}$ Yuchio Yanagawa, ${ }^{5,6}$ \\ and Tadaharu Tsumoto ${ }^{1}$ \\ ${ }^{1}$ Brain Science Institute, RIKEN, Wako 351-0198 Japan, ${ }^{2}$ Tottori University Graduate School of Medical Sciences, Yonago $683-8503$ Japan, ${ }^{3}$ Graduate School \\ of Engineering, Tokyo University of Agriculture and Technology, Koganei 184-8588 Japan, ${ }^{4}$ Department of Physiology, Hamamatsu University School of \\ Medicine, Hamamatsu 431-3192 Japan, ${ }^{5}$ Gunma University Graduate School of Medicine, Maebashi 371-8511 Japan, and ${ }^{6} J a p a n$ Science and Technology \\ Agency, Core Research for Evolutional Science and Technology, Tokyo 102-0075 Japan
}

Neuronal circuits in the cerebral cortex consist mainly of glutamatergic/excitatory and GABAergic/inhibitory neurons. In the visual cortex, the binocular responsiveness of neurons is modified by monocular visual deprivation during the critical period of postnatal development. Although GABAergic neurons are considered to play a key role in the expression of the critical period, it is not known whether their binocular responsiveness and ocular dominance plasticity are different from those of excitatory neurons. Recently, the end of the critical period was found to be not strict so that cortical neurons in the adult still have some ocular dominance plasticity. It is not known, however, which type of neurons or both maintain such plasticity in adulthood. To address these issues, we applied in vivo two-photon functional $\mathrm{Ca}^{2+}$ imaging to transgenic mice whose GABAergic neurons express a yellow fluorescent protein called Venus. We found that GABAergic neurons are more binocular than excitatory neurons in the normal visual cortex, and both types of neurons show the same degree of modifiability to monocular visual deprivation during the critical period, but the modifiability of GABAergic neurons is stronger than that of excitatory neurons after the end of the critical period.

\section{Introduction}

In the primary visual cortex, the binocular visual responsiveness of neurons can be changed by monocular visual deprivation during the critical period of postnatal development, one of the bestknown examples of experience-dependent modification of brain function (Wiesel and Hubel, 1963; Wiesel, 1982; Daw, 1995). The cerebral cortex consists mainly of two types of neurons, glutamatergic/excitatory and GABAergic/inhibitory neurons. It was proposed that GABAergic neurons play a key role in the expression of the critical period, on the basis of the results by the experimental suppression or enhancement of GABAergic function (Hensch et al., 1998) (for review, see Hensch, 2005). If GABAergic neurons play such a role in visual cortical plasticity, their binocular responsiveness and modifiability of this property to monocular visual deprivation may be different from those of excitatory neurons. It is rather surprising that the questions of whether the binocular responsiveness and experience-dependent plasticity are different between the two types of neurons remain essentially unanswered, although there was a study using glutamate decarboxylase 67 (GAD67) - green fluorescent protein (GFP) knock-in mice (Gandhi et al., 2008), in which the GABA content in the

\footnotetext{
Received 0ct. 9, 2009; revised Nov. 30, 2009; accepted Dec. 10, 2009.

We express our sincere gratitude to Dr. Atsushi Miyawaki for providing pCS2-Venus, Prof. Yoshio Hata for valuable comments to this manuscript, and Taku Uchida for assisting in part of the experiments.

*K.K. and K.S. contributed equally to this work.

Correspondence should be addressed to Dr. Tadaharu Tsumoto, Brain Science Institute, RIKEN, 2-1 Hirosawa, Wako 351-0198 Japan. E-mail: tsumoto@brain.riken.jp.

D0I:10.1523/JNEUROSC1.5025-09.2010

Copyright $\odot 2010$ the authors $\quad 0270-6474 / 10 / 301551-09 \$ 15.00 / 0$
}

brain is abnormally low because of destruction of the endogenous GAD67 gene (Tamamaki et al., 2003).

Recently, it has been found that monocular visual deprivation initiated after the end of the classically defined critical period still causes ocular dominance shift of visual cortical neurons in mice, although the magnitude, time course, and other properties of the change are not exactly the same as those of the critical period plasticity (Antonini et al., 1999; Sawtell et al., 2003; Pham et al., 2004; Tagawa et al., 2005; Hofer et al., 2006a; Fischer et al., 2007; Lehmann and Löwel, 2008; Sato and Stryker, 2008) (for review, see Hofer et al., 2006b; Morishita and Hensch, 2008). Again, it is not known which type of neurons or both maintain such plasticity in adulthood.

To answer these questions, we applied in vivo two-photon functional $\mathrm{Ca}^{2+}$ imaging to transgenic mice in which GABAergic neurons express a yellow fluorescent protein called Venus (Nagai et al., 2002) and the expression level of GAD67 in the brain is normal (Wang et al., 2009). We found that GABAergic neurons are more binocular than excitatory neurons in the normal visual cortex, and both types of neurons show the same degree of modifiability to monocular visual deprivation during the critical period, but the modifiability of GABAergic neurons is stronger than that of excitatory neurons after the end of the critical period.

\section{Materials and Methods}

Animal preparations. All experimental procedures were performed in accordance with the guidelines of the Animal Experiment Committee of RIKEN Brain Science Institute. Vesicular GABA transporter (VGAT) Venus transgenic mice that express Venus in GABAergic neurons were used. Four lines of VGAT-Venus mice were generated in the C57BL/6 
strain in the same way as VGAT-Venus rats (Uematsu et al., 2008). In the present study, we used VGAT-Venus line \#39 mice, which showed the bright fluorescence intensity (Wang et al., 2009). For monocular visual deprivation, the eyelids of the right eye (contralateral to the recording site) were sutured under anesthesia with ketamine/xylazine (100/10 $\mathrm{mg} / \mathrm{kg}$ body weight) in VGAT-Venus mice at postnatal day 27 (P27) to P28 for $2 \mathrm{~d}$. In young adult mice, the eyelid suture was made at P50-P55, when the critical period for ocular dominance plasticity is over (Gordon and Stryker, 1996), and lasted for $7 \mathrm{~d}$. The animals were returned to the cages after confirming full recovery from anesthesia. The deprivation lasted until P29-P30 ( $2 \mathrm{~d}$ of deprivation during the critical period) or until P57-P62 (7 d of deprivation after the critical period), when the imaging experiments were performed. As control, three normally reared mice at P29, P30, and P31 and another three normal mice at P59, P61, and P61 were subjected to the imaging experiments.

For the imaging experiments, the animals were anesthetized with urethane $(1.9 \mathrm{mg} / \mathrm{g}$ body weight), supplemented with additional doses $(0.25$ $\mathrm{mg} / \mathrm{g}$ ) of urethane when necessary. The animals younger than P31 were anesthetized with a half dose of urethane after a preanesthetic intraperitoneal injection of chlorprothixene $(1 \mathrm{mg} / \mathrm{kg})$. Incisions were infiltrated with lidocaine (xylocaine jelly). The level of anesthesia was continuously monitored by observing heart and respiration rates, which were not changed in response to strong touch of the tail. The animal's body temperature was maintained at $37.5^{\circ} \mathrm{C}$ by a rectal thermoprobe feeding back to a heating pad (ATC-402; Unique Medical). The part of the skull and dura mater over the primary visual cortex was removed, and the exposed cortex was covered with agarose (2-3\% in Ringer's solution). Additional details about the methods were described previously (Sohya et al., 2007).

Optical imaging of intrinsic signals. To identify the binocular region of the primary visual cortex, we adopted the fast method of optical imaging of intrinsic signals, as reported previously (Kalatsky and Stryker, 2003). Visual responses to a temporally periodic stimulus were extracted from continuously recorded images by Fourier analysis. The optical images were obtained using a CCD camera (CS8310Bi; Toshiba Teli) combined with a $50 \times 28 \mathrm{~mm}$ tandem lens (Nikon). A surface vascular image and intrinsic signal images were obtained using green $(540 \pm 10 \mathrm{~nm})$ and red $(652 \pm 10 \mathrm{~nm})$ illumination lights, respectively. The camera was focused $600 \mu \mathrm{m}$ below the pial surface at the recording session of intrinsic signals.

The periodic stimulus was generated by a visual stimulus generator (VeSaGe; Cambridge Research Systems). The bar-shaped stimulus $\left(2^{\circ}\right)$ was drifted to right or left at the spatial frequency of $75^{\circ}$ cycle and temporal frequency of $8 \mathrm{~s} / \mathrm{cycle}$. The stimulus of the drifting bar was presented to each eye. The power of intrinsic signals at the stimulus frequency was calculated for each eye, and then the binocular region of the visual cortex was visualized.

Multicell bolus dye loading and in vivo two-photon laser scanning microscopy. Bolus loading of visual cortical cells was performed with fura-2 AM (Invitrogen), as described previously (Sohya et al., 2007). A total of $10 \mathrm{~mm}$ fura-2 AM was dissolved in dimethylsulfoxide mixed with $20 \%$ Pluronic F-127 (Invitrogen) and then diluted with $0.1 \mathrm{~mm}$ sulforhodamine 101 (SR101; Invitrogen) that was dissolved in Ringer's solution. The final concentration of fura-2 AM was $0.8 \mathrm{~mm}$. A glass pipette with a tip diameter of $\sim 10 \mu \mathrm{m}$ was filled with this solution and inserted into the cortex to a depth of $200-300 \mu \mathrm{m}$ from the surface. Fura-2 AM and SR101 were ejected from the pipettes by pressure. The volume of the dye solution for each injection site and the pressure for ejection were $<1 \mathrm{pl}$ and 2-10 psi, respectively.

Images of cells stained with the dyes were visualized with an upright microscope (BX61WI; Olympus) equipped with a water-immersion objective lens (LUMPlanFL40XW; numerical aperture 0.8; Olympus) and the laser scanning microscope system (FV-1000; Olympus), which was based on the custom-made two-photon microscope system coupled with a mode-locked titanium:sapphire laser ( $<100$ fs pulse width, $80 \mathrm{MHz}$ repetition frequency at a wavelength of $800 \mathrm{~nm}$ ) (Tsunami; Spectra Physics) pumped with a $10 \mathrm{~W}$ solid-state source (Millenia PRO 10sJ 110; Spectra Physics), which was used as the excitation light source. The group velocity dispersion was compensated by prism chirp compensation, and the beam diameter for objective lens was adjusted using a Kepler telescope. The output power of the laser was adjusted by gating with an
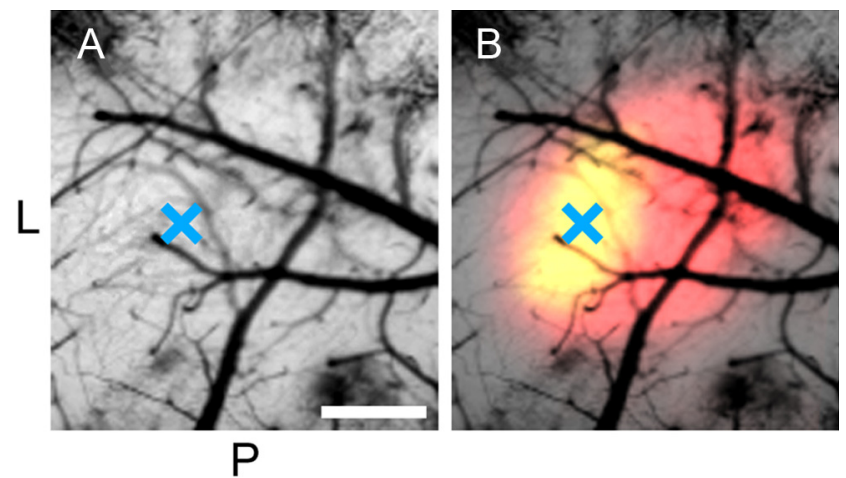

Figure 1. Optical image of mouse visual cortex. $\boldsymbol{A}$, Photograph showing the cortical surface including the primary visual cortex. L and $P$ indicate the lateral and posterior sides of the cortex, respectively. Scale bar, $0.5 \mathrm{~mm}$. The mark $X$ in the center indicates the site in which the dyes were injected. $\boldsymbol{B}$, Color-indicated map of the same area of the cortex as shown in $\boldsymbol{A}$. Areas responsive to stimulation of the contralateral and ipsilateral eyes are indicated by red and green. Green is completely overlapped with red so that the binocular region is shown as yellow.

acoustico-optic modulator. Laser power (milliwatts) measured after the objective lens was $<15 \mathrm{~mW}$ of $800 \mathrm{~nm}$ for the excitation of fura- 2 or $<60$ $\mathrm{mW}$ of $950 \mathrm{~nm}$ for the excitation of Venus and SR101 with laser irradiation time of $2 \mu \mathrm{s} /$ pixel.

Emitted fluorescence was divided into long-wavelength $(>570 \mathrm{~nm})$ and short-wavelength light with a dichroic mirror (570 nm; Olympus), and short-wavelength light was further filtered through a bandpass filter (510-550 nm; Olympus) as an emission filter. Both wavelengths of emitted fluorescence were detected simultaneously using two photomultiplier tube detectors (PMTs). The PMT offset was kept at zero throughout the recordings. The microscope objective was shielded from possible stray light by covering the space over the animal's head with lightproof cloth.

Data acquisition and analysis. For two-photon functional $\mathrm{Ca}^{2+}$ imaging, fluorescence of fura- 2 in the areas of $317 \times 317 \mu \mathrm{m}$ was imaged at $295 \mathrm{~ms} /$ frame with $512 \times 256$ pixels. After recording of neural activity, fluorescence from Venus and SR101 was simultaneously monitored by applying the above-mentioned dichroic mirrors and filters. In each animal, images were obtained from five to eight planes separated by at least $15 \mu \mathrm{m}$ at the depth of $120-230 \mu \mathrm{m}$ from the first detectable fluorescence-positive surface of the visual cortex. Time-lapse images were realigned to remove tangential drifts, and cell regions in the images were contoured visually with custom-made software. In some experiments, the images were realigned using MetaMorph software (Molecular Devices). A fura-2 signal was detected as a decrease in fluorescence intensity, which was calculated as $-\Delta F / F_{0}$, in which $\Delta F=F-F_{0}$. The baseline intensity, $F_{0}$, was obtained by averaging the intensity values during the prestimulus period ( $5 \mathrm{~s}, 16-17$ frames). The baseline signals of cells were computed by summing pixel values within the defined cell contours.

Visual stimulation and responses. Square wave gratings $\left(0.05 \mathrm{cycle} /{ }^{\circ}\right)$ were moved randomly in eight directions at four orientations (from $0^{\circ}$ to $135^{\circ}$ in $45^{\circ}$ steps) at the velocity of $20^{\circ}$ s on a liquid crystal display monitor. Four sets of these visual stimuli with an interval of $20 \mathrm{~s}$ were presented to each eye twice, and then signals for $24 \mathrm{~s}$ ( $5 \mathrm{~s}$ before, $4 \mathrm{~s}$ during, and $15 \mathrm{~s}$ after the presentation of stimuli) were averaged so that the responses for each eye were obtained. To obtain the response magnitude for each eye, the intensity values during the eight presentations of stimuli were averaged for each eye. In this averaging, negative values were regarded as zero, because they were not often observed in visually responsive cells, and even if existed they were randomly dispersed. Cells were defined as visually responsive if the responses to either eye were larger than 2 SDs of the baseline intensity during prestimulation periods.

Statistics. The Kolmogorov-Smirnov (K-S) test was used to examine significance of difference between the distributions of ocular dominance indices (ODIs). The Mann-Whitney test was used to compare values of ODIs and response magnitude between visually responsive GABAergic 

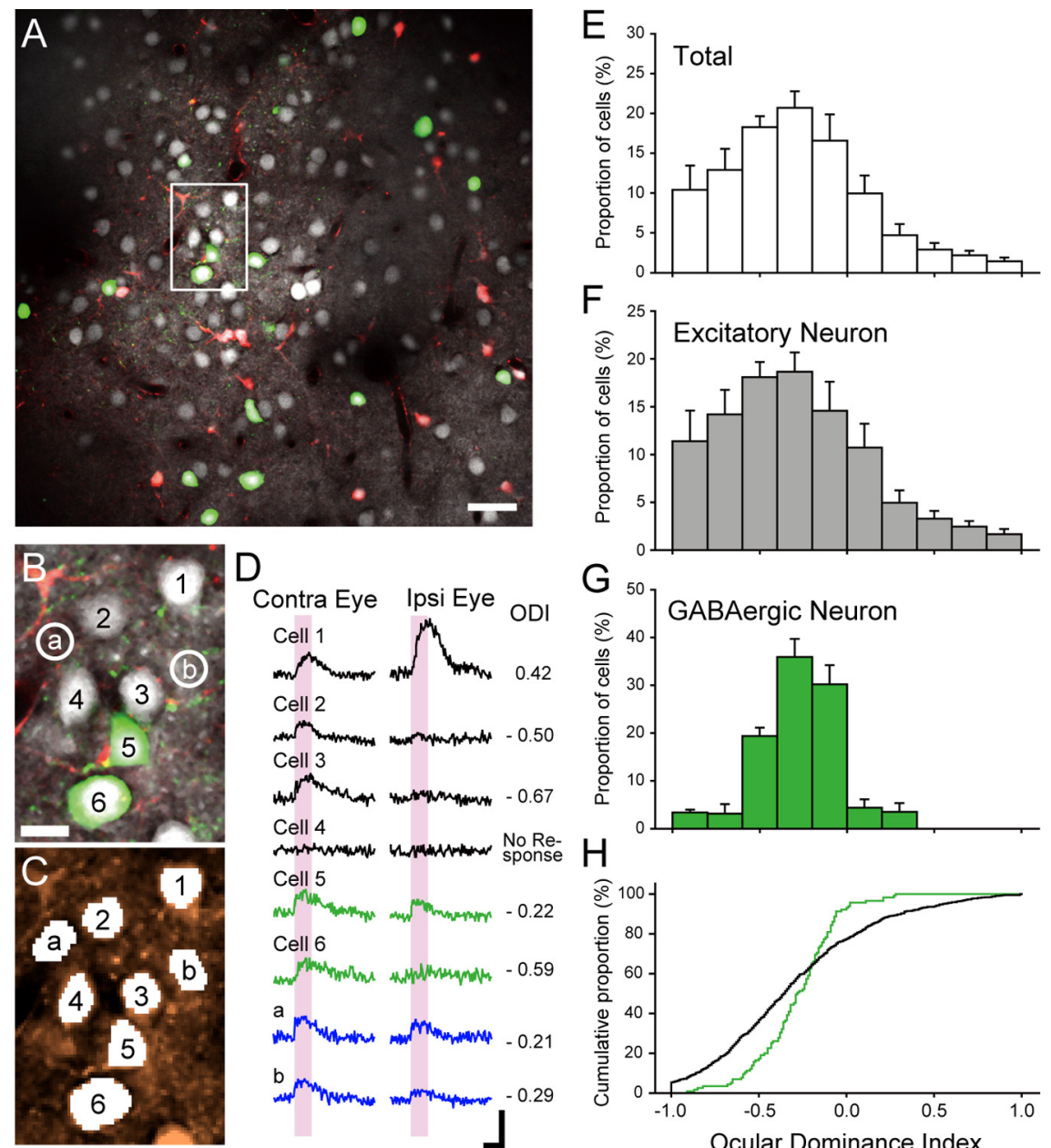

$\mathrm{F}$
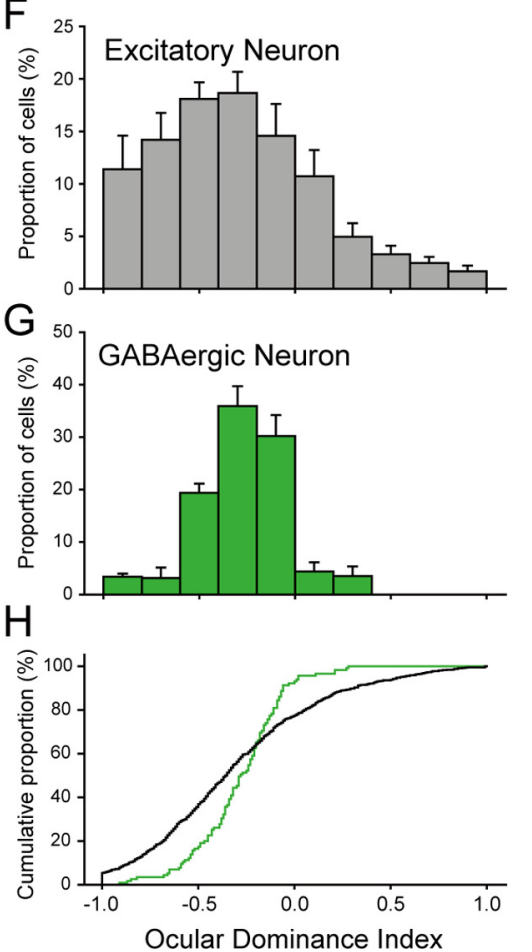

Figure 2. In vivo imaging of cells in mouse visual cortex, visual responses of excitatory and GABAergic neurons, and ocular dominance distribution of each type of neuron. $A$, Image of an optical plane of the cortex of a normally reared mouse at $\mathrm{P} 30$. This plane was obtained at the depth of $120 \mu \mathrm{m}$ from the first detected fluorescence-positive surface of the visual cortex. Scale bar, 30 $\mu \mathrm{m}$. Green, white, and red cells represent GABAergic and excitatory neurons and astrocytes, respectively. $\boldsymbol{B}$, Magnified image of the rectangular area shown in $\boldsymbol{A}$. The numbers $1-6$ indicate neurons whose responses are shown in $\boldsymbol{D}$. " $a$ " and " $b$ " indicate neuropil areas, in which responses were recorded as shown in $\boldsymbol{D}$. Scale bar, $10 \mu \mathrm{m}$. $\boldsymbol{C}$, The same area as $\boldsymbol{B}$. Regions of interest for measuring fura- 2 signals in neuronal somata and neuropil areas are shown by white areas with the same numbers and letters as in $\boldsymbol{B}$. $\boldsymbol{D}$, Traces obtained by averaging responses to visual stimulation given to the contralateral and ipsilateral eyes eight times each. Vertical columns in pink indicate the period in which visual stimulation was given. Calibration: $10 \%\left(-\Delta F / F_{0}\right), 5 \mathrm{~s}$. ODI of each cell is indicated at the right. $\boldsymbol{E}-\boldsymbol{G}, 0$ cular dominance histograms of the total of 1018 neurons, 903 excitatory neurons, and 115 GABAergic neurons, respectively, in the visual cortex of normally reared VGAT-Venus mice. Each column represents the mean proportion of cells that were rated using the 10-point scale of ODI. Error bars indicate SEM. $\boldsymbol{H}$, Cumulative distribution of the $0 \mathrm{DIs}$ of the excitatory (black line) and GABAergic (green line) neurons.

and excitatory neurons in the same rearing condition. The paired $t$ test was used to compare the monocularity indices (MIs) of GABAergic neurons with those of excitatory neurons recorded from each of the three control mice.

\section{Results}

\section{Use of VGAT-Venus mice but not GAD67-GFP}

\section{knock-in mice}

To identify GABAergic neurons in the visual cortex in the in vivo condition, we used a type of transgenic mice that express Venus, a yellow fluorescent protein, using bacterial artificial chromosome containing VGAT promoter. In these mice, $95.9 \%$ of Venus-immunoreactive cells in the cerebral cortex were GABA positive, and $93.1 \%$ of GABA-immunoreactive cells were Venus positive (Wang et al., 2009). In the present study, we did not use GAD67-GFP knock-in mice, because the expression level of GAD67 in the brain of these transgenic mice is reduced and thus the GABA content in the brain is markedly lower than that of the wild-type mice $(61 \%$ in neonates and $84 \%$ in 6- to 7-week-old mice) (Tamamaki et al., 2003). In VGATVenus mice, conversely, the expression level of GAD67 is normal (Wang et al., 2009). To confirm that the GABA content in the visual cortex of VGAT-Venus mice is normal, we measured the level of GABA in the cerebral cortex of nine transgenic and eight wild-type mice and found that the GABA level in the cortex was not significantly different between these two types of mice (supplemental Fig. 1, available at www.jneurosci.org as supplemental material).

\section{Identification of GABAergic and excitatory neurons in binocular region} To identify the binocular region of the primary visual cortex, we used optical imaging of cortical intrinsic signals evoked by visual stimuli given to the contralateral and ipsilateral eyes (Grinvald et al., 1986; Kalatsky and Stryker, 2003; Cang et al., 2005). With optical imaging, we could identify the binocular region of the primary visual cortex as the cortical area, in which responses to ipsilateral as well as contralateral eye stimulation were induced (Fig. 1). In Figure $1 B$, the areas in which responses to the contralateral and ipsilateral eyes were obtained are colored in red and green, respectively, so that the binocular region is shown in yellow. The mark $\mathrm{X}$ in the center indicates the site in which the dyes were injected. Thus, all the neurons in the present report were recorded from the binocular region of the primary visual cortex of VGAT-Venus mice.

Then, the bolus loading of visual cortical cells was performed with fura-2 AM and SR101, as described previously (Sohya et al., 2007). Using these dyes and the twophoton laser scanning microscope system, we could differentially measure the responses of GABAergic and excitatory neurons to visual stimuli given to each eye (Fig. $2 A, B$ ) (supplemental Fig. 2, available at www.jneurosci.org as supplemental material). Astrocytes stained with SR101 were excluded from the present analysis. As shown in Figure $2 D$, stained neurons showed clear visual responses to both eyes or either eye, although some neurons did not generate significant responses. We also observed responses in neuropil areas surrounding the somata of the neurons. The area sizes for measurements of neuropil signals were set to be nearly the same as those for neuronal somata (Fig. 2C). In Figure 2D, it is seen that the neuropil areas "a" and "b" showed responses to ipsilateral as well as contralateral eye stimulation, whereas the nearby somata of the cells 2 and 3 did not show clear responses to ipsilateral eye stimulation, indicating that neuropil signals did not notably contribute to the signals of neuronal somata, as reported previously (Sohya et al., 2007). In 17 planes of the three normal mice at 
P29-P31, 903 (43\%) of 2091 excitatory neurons and 115 (51\%) of 224 GABAergic neurons showed significant responses to visual stimuli. These proportions of visually responsive neurons are similar to those obtained previously in GAD67GFP mice (34 and 40\%, respectively) (Sohya et al., 2007) and that of unclassified neurons in the rat visual cortex $(37 \%)$ (Ohki et al., 2005).

GABAergic neurons are more binocular than excitatory neurons

To quantify the preference of visual responses of cortical neurons to either eye, we calculated the ODI of each neuron, as follows:

$$
\mathrm{ODI}=\left(R_{\mathrm{I}}-R_{\mathrm{C}}\right) /\left(R_{\mathrm{I}}+R_{\mathrm{C}}\right),
$$

where $R_{\mathrm{I}}$ is the magnitude of responses to the ipsilateral (left) eye (open eye in the case of monocular deprivation), and $R_{\mathrm{C}}$ is the response magnitude to the contralateral (right) eye (deprived eye). To show the distribution of ODI as an ocular dominance histogram, each neuron was rated using a 10-point scale. In the three normally reared mice at P29-P31, the distributions of ODIs of the 1018 neurons were biased toward the contralateral eye (Fig. $2 E)$, as reported previously in the visual cortex of wild-type mice (Dräger, 1975; Métin et al., 1988; Gordon and Stryker, 1996). When neurons were classified into the two groups, nonGABAergic (excitatory) and GABAergic (inhibitory), the distributions of ODIs of the 903 excitatory neurons were also biased toward the contralateral eye (Fig. $2 F$ ). The mean ODI of these excitatory neurons was $-0.303 \pm 0.015$ (SEM) (see Fig. 6). Different from that of the excitatory neurons, the ODI distribution of the 115 GABAergic neurons was concentrated to the binocular value ranging mostly from 0.0 to -0.6 (Fig. $2 G$ ). The mean ODI of the GABAergic neurons was $-0.282 \pm 0.021$ (see Fig. 6). Although this mean value seemed to be not different from that of excitatory neurons, the analysis with the cumulative proportion of the excitatory and GABAergic neurons showed a significant ( $\mathrm{K}-\mathrm{S}$ test, $p<0.001$ ) difference in the ODI distribution between the two types of neurons (Fig. $2 H$ ). The same line of results as those mentioned above were obtained from the three normally reared mice at P59-P61 (supplemental Fig. 3, available at www. jneurosci.org as supplemental material). The ODI distribution of the 365 GABAergic neurons of these normal mice was not significantly different from that of the 115 GABAergic neurons of the mice at P29-P31 (K-S test, $p=0.418$ ).

To quantify the difference in binocular visual responsiveness between excitatory and GABAergic neurons, we calculated the MI for each mouse (Fig. 3). MI was calculated as follows:

$$
\begin{aligned}
\mathrm{MI}=\left[\left(N_{1}+N_{10}\right)+\left(N_{2}+N_{9}\right) \times 3 / 4\right. & +\left(N_{3}+N_{8}\right) \times 2 / 4 \\
& \left.+\left(N_{4}+N_{7}\right) \times 1 / 4\right] / N_{T},
\end{aligned}
$$

where $N_{n}$ is the number of cells classified as group $n$ using the 10 -point scale of ODI, and $N_{T}$ is the total number of visually responsive cells. For example, groups 1 and 10 consisted of neurons with ODIs of less than -0.8 to -1.0 and $>0.8$ to 1.0 , respectively. Groups 2-9 consisted of neurons with ODIs of intermediate values in 0.2 steps. If all the visually responsive cells are classified as monocular, MI will be 1 . If all the cells are rated as group 5 or 6 , MI will be 0 . We found that the mean MI of the excitatory neurons for the three normal mice at P29-P31 $(0.421 \pm 0.057)$ was significantly (paired $t$ test, $p<0.05)$ larger than that $(0.253 \pm 0.021)$ of the GABAergic neurons. Such a difference in the mean MI between the two types of neurons was seen also in the three normal mice at P59-P61 (0.368 \pm 0.060 for excitatory neurons and $0.271 \pm 0.069$ for GABAergic neurons; paired $t$ test, $p<0.05)$. These results indicate that GABAergic neurons are more binocular than excitatory neurons in the visual cortex of normally reared mice.

\section{Ocular dominance shift of both types of neurons during the critical period}

In the three mice that were monocularly deprived for $2 \mathrm{~d}$ starting at P27-P28 during the critical period, we found that the deprivation-induced shift in ocular dominance took place in both excitatory and GABAergic neurons (Fig. 4). In 445 visually responsive cells of 1081 excitatory neurons, the mean ODI of the responsive cells was $-0.076 \pm 0.022$ (Fig. $4 A$, columns with solid lines) (see also Fig. 6). This ODI distribution of the visually responsive excitatory neurons was different from that of the normal mice (Fig. $2 \mathrm{~F}$; shown as columns with broken lines in Fig. 4A). The cumulative analysis confirmed that the two distributions are significantly (K-S test, $p<10^{-9}$ ) different (Fig. $4 B$ ). In 114 visually responsive cells of 181 GABAergic neurons of the mice 

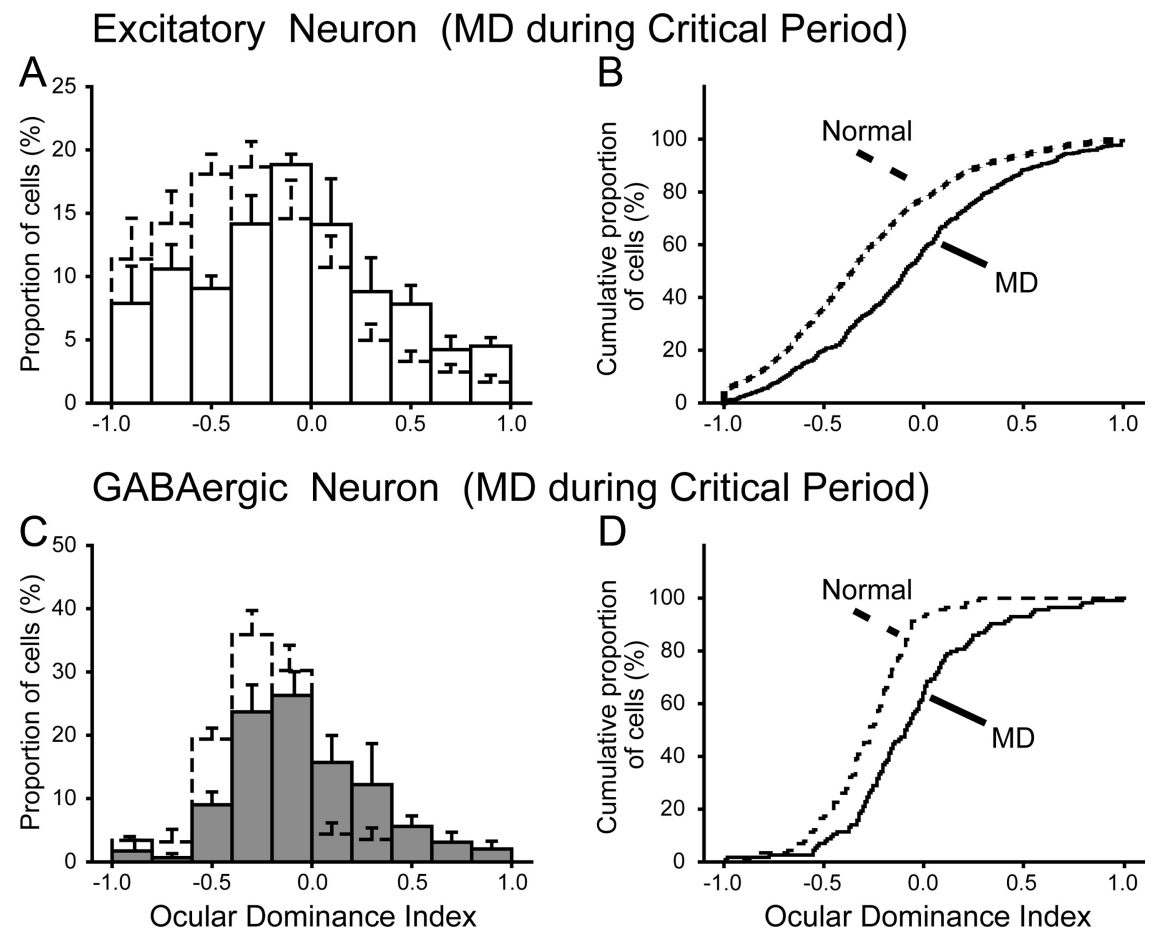

Figure 4. Ocular dominance histograms and cumulative distribution of ODls obtained from the mice that were monocularly deprived for $2 \mathrm{~d}$ during the critical period. $A$, Ocular dominance histograms of 445 excitatory neurons obtained from the three deprived mice. Columns and bars with broken lines indicate the data shown in Figure $2 F$. Other conventions are the same as in Figure $2 E-G . B$, Cumulative distributions of the $0 D I s$ of the excitatory neurons of the deprived (solid line) and normal (broken line) mice. MD, Monocular visual deprivation. $C$, Ocular dominance histograms of 114 GABAergic neurons obtained from the three deprived mice. Columns and bars with broken lines indicate the data shown in Figure 2G.D. Cumulative distributions of the ODIs of the GABAergic neurons of the deprived (solid line) and normal (broken line) mice.

that were monocularly deprived during the critical period, the mean ODI of the responsive cells was $-0.068 \pm 0.032$ (Fig. $4 C$, filled columns) (see also Fig. 6). The ODI distribution of these GABAergic neurons was different from that of the 115 GABAergic neurons of the normal mice (Fig. 2G; shown as open columns with broken lines in Fig. 4C). The cumulative analysis also showed a significantly ( $\mathrm{K}-\mathrm{S}$ test, $p<10^{-4}$ ) different distribution between the two groups of GABAergic neurons (Fig. $4 D$ ). It is to be noted that, in the mice with monocular deprivation during the critical period, the extent of the ODI shift of the GABAergic neurons was almost the same as that of the excitatory neurons (see Fig. 6).

\section{Stronger effect of deprivation on GABAergic neurons after the critical period}

In the mouse, the critical period for ocular dominance plasticity of visual cortical neurons was reported to be P19-P32 (Gordon and Stryker, 1996). Recently, however, it has been found that visual cortical neurons still show a certain type of plasticity after the end of the critical period, as mentioned in Introduction. In this study, we tested whether monocular deprivation for $7 \mathrm{~d}$ after the critical period can induce a shift of ODIs in excitatory or GABAergic neurons or both. We found that both types of neurons showed such a shift, but the change seen in GABAergic neurons was stronger than that in excitatory neurons (Fig. $5 A, C$ ) (supplemental Fig. $2 D$, available at www.jneurosci.org as supplemental material). The mean ODI of 1266 visually responsive cells of 2694 excitatory neurons recorded from the three deprived mice at P57, P58, and P62 was $-0.215 \pm 0.012$ (see Fig. 6, open circle in the rightmost group). The corresponding value of 1529 visually responsive cells of 2903 excitatory neurons recorded from the three normal mice at P59, P61, and P61 was $-0.295 \pm$ 0.010 (Fig. 6, open circle in the third group). The cumulative analysis indicated that the distribution of the visually responsive excitatory neurons was significantly (K-S test, $p<10^{-4}$ ) different from that of the normal mice (Fig. $5 B$ ). Also, GABAergic neurons showed a clear shift of ODIs toward the open eye (Fig. 5C). The mean ODI of 128 visually responsive cells of 294 GABAergic neurons obtained from the deprived mice was $-0.121 \pm$ 0.027 (Fig. 6, filled circle in the rightmost group). The mean value of 182 visually responsive cells of 365 GABAergic neurons recorded from the three age-matched control mice was $-0.273 \pm 0.022$ (Fig. 6, filled circle in the third group). The cumulative analysis confirmed a significant $(\mathrm{K}-\mathrm{S}$ test, $p<10^{-6}$ ) difference in the ODI distribution between the two groups of GABAergic neurons (Fig. 5D).

To show the changes in ODI more clearly, the mean values for excitatory and GABAergic neurons in the four groups of mice are plotted in Figure 6. In the mice that were monocularly deprived after the end of the critical period, the ODI of the GABAergic neurons was significantly (Mann-Whitney test, $p<10^{-4}$ ) larger than that of the excitatory neurons (rightmost group) compared with no difference (Mann-Whitney test, $p>0.1)$ in the mice that were deprived during the critical period (second group). To test whether a possible contamination with neuropil signals might affect the difference in the ODI between the two types of neurons after the critical period, we also plotted the ODIs of neuropil signals in Figure 6 (open squares). The mean value of the neuropil signals in the mice deprived after the critical period was close to that of excitatory neurons but significantly different from that of GABAergic neurons (Fig. 6, rightmost group), indicating that a possible contamination with neuropil signals if it existed did not notably contribute to the difference in the ODI between excitatory and GABAergic neurons after the critical period. Thus, these results altogether suggest that GABAergic neurons were affected more strongly by monocular visual deprivation after the end of the critical period than excitatory neurons.

\section{Difference in the deprivation-induced change in the response} magnitude between GABAergic and excitatory neurons

To examine reasons for the difference in the degree of the ODI shift between the two types of neurons in the mice deprived after the critical period, we measured the magnitude of visual responses to each eye (Fig. 7A). The magnitude of responses of the GABAergic neurons to the closed (contralateral) eye of the deprived mice was significantly (Mann-Whitney test, $p<0.01$ ) smaller than that to the normal (contralateral) eye of the normally reared mice (Fig. $7 A b$, left two columns), whereas that of the excitatory neurons was not significantly (Mann-Whitney test, $p>0.1$ ) changed (Fig. 7Aa, left two columns). The response of the excitatory neurons to the open (ipsilateral) eye of the de- 
prived mice was slightly but significantly (Mann-Whitney test, $p<10^{-4}$ ) enhanced (Fig. 7Aa, right two columns), whereas that of the GABAergic neurons was not changed (Mann-Whitney test, $p>0.05$ ) (Fig. 7Ab, right two columns). Thus, the stronger ODI shift of GABAergic neurons after the critical period may be ascribable to the strong depression of visual responses to the closed eye, whereas the weaker ODI shift of excitatory neurons may be attributable to the weak potentiation of responses to the open eye.

We also analyzed the magnitude of visual responses to each eye in the mice that were deprived during the critical period (Fig. $7 B$ ). In both types of neurons, the magnitudes of responses to the open (ipsilateral) eye were significantly (Mann-Whitney test, $p<0.01$ ) larger than those to the normal (ipsilateral) eye of the control mice (Fig. $7 B a, B b$, right two columns, respectively). Conversely, the magnitude of responses of the excitatory neurons to the closed (contralateral) eye was significantly (Mann-Whitney test, $p<0.01)$ smaller than that to the normal (contralateral) eye of the control mice (Fig. $7 \mathrm{Ba}$, left two columns), whereas that of the GABAergic neurons was not significantly changed (Mann-Whitney test, $p>0.1$ ) (Fig. 7Bb, left two columns).

\section{Discussion}

\section{Binocularity of GABAergic neurons}

In this study, we found that the distribution of ODIs of GABAergic neurons in the visual cortex of normally reared mice was restricted mostly within the binocular range, whereas that of excitatory neurons was more widespread, although the mean ODIs were not significantly different. Recently, it has been reported that both types of neurons showed the similar distribution of ODIs in GAD67-GFP knock-in mice at P25, P28, P30, and P35 (Gandhi et al., 2008). This seemingly different result from the present study may reflect the reduction in the GABA content in the brain of GAD67-GFP knock-in mice, although other factors such as different anesthetics are not completely excluded.

The GABA content in the brain of GAD67-GFP knock-in mice is significantly lower than that of the wild-type mice $(61 \%$ in neonates and $84 \%$ in 6- to 7 -week-old mice) (Tamamaki et al., 2003). A previous study showed that an intracortical application of a GABA antagonist reduced the monocularity of responses of visual cortical neurons in the cat, suggesting that the intracortical GABAergic inhibition makes visual responses of cortical neurons more monocular (Sillito et al., 1980). In the visual cortex, pyramidal cells receive very powerful GABAergic inhibition from adjacent basket cells, the most popular type of GABAergic neurons in the visual cortex, although basket cells themselves also receive some inhibitory inputs (Kisvárday, 1992; Somogyi et al., 1998) (for review, see Markram et al., 2004). In the visual cortex with low GABA content, therefore, the ocular dominance distribution of excitatory neurons might become more binocular than in the normal cortex so that the difference between excitatory and GABAergic neurons is undetectable. In the previous study (Gandhi et al., 2008), in fact, the mean ODI value of the excitatory neurons $(0.23 \pm 0.01$, which corresponds to $-0.23 \pm 0.01$ with our equation) seems to be closer to zero than that of our excitatory neurons $(-0.303 \pm 0.015)$.

The more binocular responsiveness of GABAergic neurons than excitatory neurons may be ascribable in part to the wide dendritic trees of basket cells in the visual cortex (Feldman and Peters, 1978; Markram et al., 2004; Kawaguchi et al., 2006) and/or to the abundance of reciprocal connections between GABAergic and pyramidal neurons in layer $2 / 3$ of the visual cortex (Somogyi et al., 1998; Yoshimura and Callaway, 2005). In this arrangement of circuits, GABAergic neurons may receive excitatory, driving inputs from adjacent pyramidal neurons, whereas pyramidal neurons receive inhibitory inputs from GABAergic neurons so that the spike-generating responses of GABAergic neurons tend to be more widespread and those of pyramidal cells tend to be more restricted.

It is suggested that the activity-dependent modifiability of ocular dominance of visual cortical neurons is controlled by the functional maturation of the GABAergic inhibitory system in the cortex (Hensch et al., 1998; Hensch, 2005). The strong binocularity of GABAergic neurons may be a basis for such a role, because it provides a place in which binocular interaction operates. 


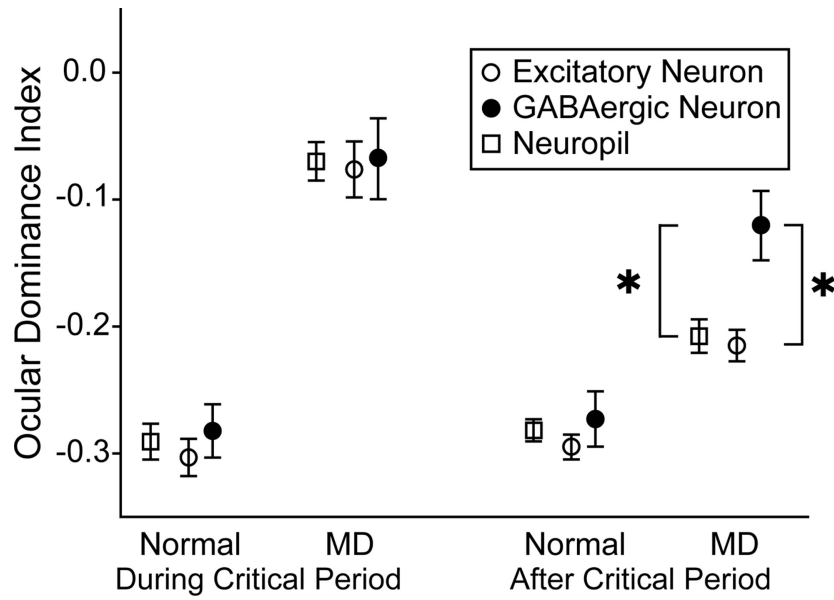

Figure 6. Mean ODIs of excitatory (open circles) and GABAergic (filled circles) neurons obtained from four different groups of mice. The values of neuropil signals were also included (open squares). Vertical bars indicate SEMs. Mean values for the 903 excitatory and $115 \mathrm{GABAer}-$ gic neurons of the normally reared mice at the age during the critical period were $-0.303 \pm$ 0.015 (SEM) and $-0.282 \pm 0.021$, respectively. Those for the 445 excitatory and 114 GABAergic neurons in the deprived mice during the critical period were $-0.076 \pm 0.022$ and $-0.068 \pm 0.032$, respectively. Mean values for the 1529 excitatory and 182 GABAergic neurons of the normally reared mice at the age after the critical period were $-0.295 \pm 0.010$ and $-0.273 \pm 0.022$, respectively, and those for the 1266 excitatory and $128 \mathrm{GABAergic}$ neurons in the deprived mice after the critical period were $-0.215 \pm 0.012$ and $-0.121 \pm 0.027$, respectively. Mean values of neuropil signals of the four groups of mice from the leftmost to rightmost columns were $-0.291 \pm 0.014(n=180),-0.070 \pm 0.015(n=175)$, $-0.282 \pm 0.009(n=268)$, and $-0.208 \pm 0.013(n=255)$, respectively. ${ }^{*} p<10^{-4}$, Mann-Whitney test. MD, Monocular visual deprivation.

\section{Ocular dominance shift during the critical period}

Regarding the effects of monocular deprivation during the critical period, it is known that deprivation for just $2-3 \mathrm{~d}$ leads to a significant shift of binocular visual responses of nonclassified neurons to the open eye in the visual cortex of wild-type mice (Mrsic-Flogel et al., 2007), although another previous study reported that deprivation not for $2 \mathrm{~d}$ but for $4 \mathrm{~d}$ was necessary to induce the maximum effect (Gordon and Stryker, 1996). In GAD67-GFP knock-in mice, it was reported that the ODI of GABAergic neurons was little changed after $2 \mathrm{~d}$ of monocular deprivation during the critical period, but that of excitatory neurons was significantly shifted toward the open eye (Gandhi et al., 2008). In the present study, we found that the significant shift in ocular dominance occurs in both excitatory and GABAergic neurons after monocular deprivation for $2 \mathrm{~d}$ during the critical period. This difference may be ascribable to the low GABA content in the brain of GAD67-GFP knock-in mice or to other factors such as anesthesia, as mentioned above.

In intracellular recordings from visual cortical neurons of wild-type mice, it has been reported that fast-spiking interneurons exhibited a counterintuitive shift of spike responses toward the deprived eye after short-term deprivation $(2-3 \mathrm{~d})$ and then shifted toward the open eye after long-term deprivation $(>2$ weeks) (Yazaki-Sugiyama et al., 2009). In the present study, we did not observe such a shift after $2 \mathrm{~d}$ deprivation. This might be ascribable at least in part to our sampling of GABAergic neurons, which probably included non-fast-spiking as well as fast-spiking neurons. There are other possible factors for this discrepancy: intracellular recordings from a limited number of cells under pentobarbital anesthesia versus functional calcium imaging of many cells under urethane anesthesia.

\section{A After Critical Period} a Excitatory Neurons

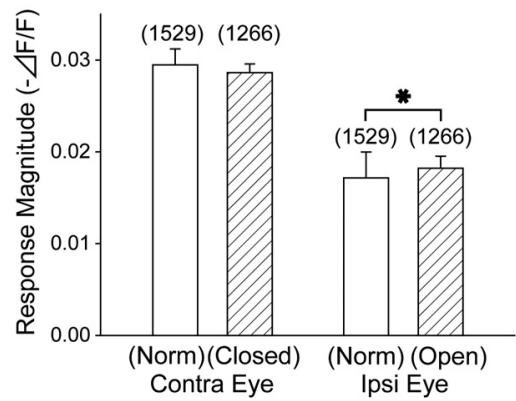

B During Critical Period a Excitatory Neurons

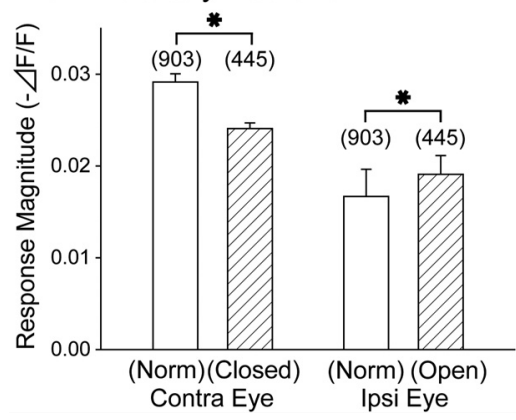

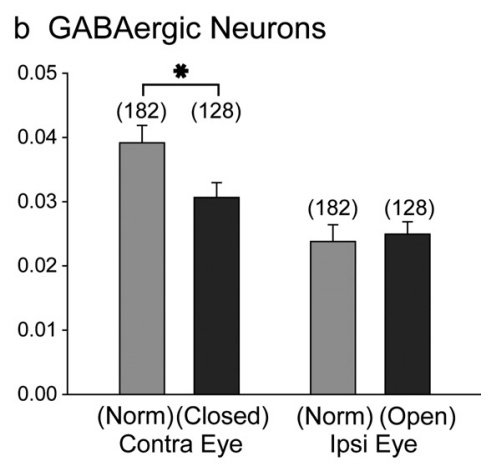

b GABAergic Neurons

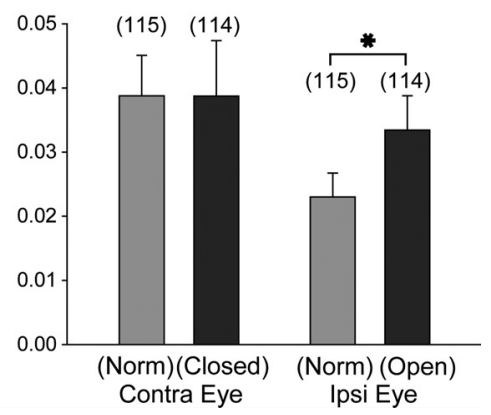

Figure 7. Magnitude of visual responses of excitatory (left) and GABAergic (right) neurons recorded from the monocularly deprived and age-matched normal mice after $(\boldsymbol{A})$ and during $(\boldsymbol{B})$ the critical period. Each column represents means of median response magnitudes of individual animals for each group of neurons as shown at the abscissa. Error bars indicate SEMs. Numbers in parentheses indicate number of neurons in each group. Asterisks in $\boldsymbol{A}$ and $\boldsymbol{B}$ indicate that the difference was statistically significant (see Results).

Susceptibility of GABAergic neurons to monocular deprivation after the critical period

Recently, it has been reported that monocular visual deprivation initiated after the end of the classically defined critical period causes ocular dominance shift of visual cortical neurons in young adult mice (see Introduction). The present study demonstrated for the first time that the extent to which binocular responsiveness is changed in response to monocular visual deprivation after the end of the critical period is stronger in GABAergic neurons than in excitatory neurons. Furthermore, the change in ODI of the GABAergic neurons is accompanied by the depression of visual responses to the deprived eye. These results seem to be in line with the previous observations that monocular deprivation results in the reduction of immunoreactivity for GABA and its synthesizing enzyme GAD in input-receiving layers of the primary visual cortex of adult monkeys (Hendry and Jones, 1986), cats (Rosier et al., 1995), and rats (Benevento et al., 1995).

\section{Difference in plasticity between} GABAergic and excitatory neurons

The present results demonstrated that the responses of GABAergic neurons to the 
closed eye were depressed by monocular deprivation after the end of the critical period. This may be accounted for by the Hebbian form of synaptic plasticity, as conventionally thought to operate in the visual cortex (Bienenstock et al., 1982; Miller et al., 1989; Daw, 1995; Bear, 2003). The present results also showed that the open-eye responses of excitatory neurons were potentiated by monocular deprivation after the critical period. This seems consistent with the previous results that the responses of visual cortical neurons to the open eye were enhanced after monocular deprivation in young adult mice, although neurons were not classified into the two types (Sawtell et al., 2003; Frenkel et al., 2006; Hofer et al., 2006a; Sato and Stryker, 2008). The above-mentioned depression of responses of GABAergic neurons might play a permissive role in this potentiation of the open-eye responses of excitatory neurons.

The present study also showed that the responses of excitatory neurons to the deprived eye were not significantly depressed. Such an apparent resistance to deprivation-induced change might reflect the homeostatic plasticity of cortical synapses, as suggested previously (Bear, 2003; Turrigiano and Nelson, 2004; Mrsic-Flogel et al., 2007; Kaneko et al., 2008). The present findings further suggest that the operation of such homeostatic plasticity in the deprived-eye responses of excitatory neurons may be facilitated by or cooperated with the depression of the responsiveness of GABAergic neurons.

During the critical period, the responses of the excitatory neurons to the closed eye were depressed, but those of the GABAergic neurons were not significantly changed. The depression of the excitatory neurons is consistent with the previous results (Gandhi et al., 2008). No change in the response magnitude of GABAergic neurons might result from a mixture of enhancement of fast-spiking neurons (Yazaki-Sugiyama et al., 2009) and depression of nonfast-spiking neurons. During the critical period, the responses to the open eye were enhanced in both types of neurons. This is consistent with the 7-10 d deprivation effect but not with the $2 \mathrm{~d}$ deprivation effect in the previous report (Gandhi et al., 2008). This inconsistency may result from the low GABA content of GAD67-GFP mice that might make the enhancement of the open-eye response slow.

\section{Limitation of cell sampling to the superficial layers of the cortex}

Because of the limitation of the present two-photon laser scanning system, we recorded cells located up to $230 \mu \mathrm{m}$ deep from the first detected fluorescence-positive surface of the cortex. Thus, all the cells analyzed in the present study were located in layer $2 / 3$ of the primary visual cortex. Previously, it was reported that the effect of monocular deprivation starts and terminates earlier in layer 4 of the visual cortex than in other layers, and the ocular dominance plasticity in layer 2/3 remains after the critical period to a substantial extent in juvenile animals (Daw et al., 1992; Guire et al., 1999; Sawtell et al., 2003; Pham et al., 2004; Sato and Stryker, 2008). Also, it was reported that ocular dominance plasticity in layer $2 / 3$ is more vigorous than that in layer 4 of the mouse visual cortex during the critical period (Gordon and Stryker, 1996). Therefore, it may not be appropriate to simply extend the present results obtained from layer $2 / 3$ to the other layers of the visual cortex.

\section{References}

Antonini A, Fagiolini M, Stryker MP (1999) Anatomical correlates of functional plasticity in mouse visual cortex. J Neurosci 19:4388-4406.

Bear MF (2003) Bidirectional synaptic plasticity: from theory to reality. Philos Trans R Soc Lond B Biol Sci 358:649-655.
Benevento LA, Bakkum BW, Cohen RS (1995) Gamma-aminobutyric acid and somatostatin immunoreactivity in the visual cortex of normal and dark-reared rats. Brain Res 689:172-182.

Bienenstock EL, Cooper LN, Munro PW (1982) Theory for the development of neuron selectivity: orientation specificity and binocular interaction in visual cortex. J Neurosci 2:32-48.

Cang J, Kalatsky VA, Löwel S, Stryker MP (2005) Optical imaging of the intrinsic signal as a measure of cortical plasticity in the mouse. Vis Neurosci 22:685-691.

Daw NW (1995) Visual development. New York: Plenum.

Daw NW, Fox K, Sato H, Czepita D (1992) Critical period for monocular deprivation in the cat visual cortex. J Neurophysiol 67:197-202.

Dräger UC (1975) Receptive fields of single cells and topography in mouse visual cortex. J Comp Neurol 160:269-290.

Feldman ML, Peters A (1978) The forms of non-pyramidal neurons in the visual cortex of the rat. J Comp Neurol 179:761-793.

Fischer QS, Graves A, Evans S, Lickey ME, Pham TA (2007) Monocular deprivation in adult mice alters visual acuity and single-unit activity. Learn Mem 14:277-286.

Frenkel MY, Sawtell NB, Diogo AC, Yoon B, Neve RL, Bear MF (2006) Instructive effect of visual experience in mouse visual cortex. Neuron 51:339-349.

Gandhi SP, Yanagawa Y, Stryker MP (2008) Delayed plasticity of inhibitory neurons in developing visual cortex. Proc Natl Acad Sci U S A 105:16797-16802.

Gordon JA, Stryker MP (1996) Experience-dependent plasticity of binocular responses in the primary visual cortex of the mouse. J Neurosci 16:3274-3286.

Grinvald A, Lieke E, Frostig RD, Gilbert CD, Wiesel TN (1986) Functional architecture of cortex revealed by optical imaging of intrinsic signals. Nature 324:361-364.

Guire ES, Lickey ME, Gordon B (1999) Critical period for the monocular deprivation effect in rats: assessment with sweep visually evoked potentials. J Neurophysiol 81:121-128.

Hendry SH, Jones EG (1986) Reduction in number of immunostained GABAergic neurons in deprived-eye dominance columns of monkey area 17. Nature 320:750-753.

Hensch TK (2005) Critical period plasticity in local cortical circuits. Nat Rev Neurosci 6:877-888.

Hensch TK, Fagiolini M, Mataga N, Stryker MP, Baekkeskov S, Kash SF (1998) Local GABA circuit control of experience-dependent plasticity in developing visual cortex. Science 282:1504-1508.

Hofer SB, Mrsic-Flogel TD, Bonhoeffer T, Hübener M (2006a) Prior experience enhances plasticity in adult visual cortex. Nat Neurosci 9:127-132.

Hofer SB, Mrsic-Flogel TD, Bonhoeffer T, Hübener M (2006b) Lifelong learning: ocular dominance plasticity in mouse visual cortex. Curr Opin Neurobiol 16:451-459.

Kalatsky VA, Stryker MP (2003) New paradigm for optical imaging: temporally encoded maps of intrinsic signal. Neuron 38:529-545.

Kaneko M, Stellwagen D, Malenka RC, Stryker MP (2008) Tumor necrosis factor- $\alpha$ mediates one component of competitive, experience-dependent plasticity in developing visual cortex. Neuron 58:673-680.

Kawaguchi Y, Karube F, Kubota Y (2006) Dendritic branch typing and spine expression patterns in cortical nonpyramidal cells. Cereb Cortex 16:696-711.

Kisvárday ZF (1992) GABAergic networks of basket cells in the visual cortex. Prog Brain Res 90:385-405.

Lehmann K, Löwel S (2008) Age-dependent ocular dominance plasticity in adult mice. PLoS ONE 3:e3120.

Markram H, Toledo-Rodriguez M, Wang Y, Gupta A, Silberberg G, Wu C (2004) Interneurons of the neocortical inhibitory system. Nat Rev Neurosci 5:793-807.

Métin C, Godement P, Imbert M (1988) The primary visual cortex in the mouse: receptive field properties and functional organization. Exp Brain Res 69:594-612.

Miller KD, Keller JB, Stryker MP (1989) Ocular dominance column development: analysis and simulation. Science 245:605-615.

Morishita H, Hensch TK (2008) Critical period revisited: impact on vision. Curr Opin Neurobiol 18:101-107.

Mrsic-Flogel TD, Hofer SB, Ohki K, Reid RC, Bonhoeffer T, Hübener M (2007) Homeostatic regulation of eye-specific responses in visual cortex during ocular dominance plasticity. Neuron 54:961-972. 
Nagai T, Ibata K, Park ES, Kubota M, Mikoshiba K, Miyawaki A (2002) A variant of yellow fluorescent protein with fast and efficient maturation for cell-biological applications. Nat Biotechnol 20:87-90.

Ohki K, Chung S, Ch'ng YH, Kara P, Reid RC (2005) Functional imaging with cellular resolution reveals precise micro-architecture in visual cortex. Nature 433:597-603.

Pham TA, Graham SJ, Suzuki S, Barco A, Kandel ER, Gordon B, Lickey ME (2004) A semi-persistent adult ocular dominance plasticity in visual cortex is stabilized by activated CREB. Learn Mem 11:738-747.

Rosier AM, Arckens L, Demeulemeester H, Orban GA, Eysel UT, Wu YJ, Vandesande F (1995) Effect of sensory deafferentation on immunoreacitivity of GABAergic cells and on GABA receptors in the adult cat visual cortex. J Comp Neurol 359:476-489.

Sato M, Stryker MP (2008) Distinctive features of adult ocular dominance plasticity. J Neurosci 28:10278-10286.

Sawtell NB, Frenkel MY, Philpot BD, Nakazawa K, Tonegawa S, Bear MF (2003) NMDA receptor-dependent ocular dominance plasticity in adult visual cortex. Neuron 38:977-985.

Sillito AM, Kemp JA, Patel H (1980) Inhibitory interactions contributing to the ocular dominance of monocularly dominated cells in the normal cat striate cortex. Exp Brain Res 41:1-10.

Sohya K, Kameyama K, Yanagawa Y, Obata K, Tsumoto T (2007) GABAergic neurons are less selective to stimulus orientation than excitatory neurons in layer II/III of visual cortex, as revealed by in vivo functional $\mathrm{Ca}^{2+}$ imaging in transgenic mice. J Neurosci 27:2145-2149.

Somogyi P, Tamás G, Lujan R, Buhl EH (1998) Salient feature of synaptic organization in the cerebral cortex. Brain Res Rev 26:113-135.
Tagawa Y, Kanold PO, Majdan M, Shatz CJ (2005) Multiple periods of functional ocular dominance plasticity in mouse visual cortex. Nat Neurosci $8: 380-388$.

Tamamaki N, Yanagawa Y, Tomioka R, Miyazaki J, Obata K, Kaneko T (2003) Green fluorescent protein expression and colocalization with calretinin, parvalbumin, and somatostatin in the GAD67-GFP knock-in mouse. J Comp Neurol 467:60-79.

Turrigiano GG, Nelson SB (2004) Homeostatic plasticity in the developing nervous system. Nat Rev Neurosci 5:97-107.

Uematsu M, Hirai Y, Karube F, Ebihara S, Kato M, Abe K, Obata K, Yoshida S, Hirabayashi M, Yanagawa Y, Kawaguchi Y (2008) Quantitative chemical composition of cortical GABAergic neurons revealed in transgenic Venus-expressing rats. Cereb Cortex 18:315-330.

Wang Y, Kakizaki T, Sakagami H, Saito K, Ebihara S, Kato M, Hirabayashi M, Saito Y, Furuya N, Yanagawa Y (2009) Fluorescent labeling of both GABAergic and glycinergic neurons in vesicular GABA transporter (VGAT)-Venus transgenic mouse. Neuroscience 164:1031-1043.

Wiesel TN (1982) Postnatal development of the visual cortex and the influence of environment. Nature 299:583-591.

Wiesel TN, Hubel DH (1963) Single-cell responses in striate cortex of kittens deprived of vision in one eye. J Neurophysiol 26:1003-1017.

Yazaki-Sugiyama Y, Kang S, Câteau H, Fukai T, Hensch TK (2009) Bidirectional plasticity in fast-spiking GABA circuits by visual experience. Nature 462:218-221.

Yoshimura Y, Callaway EM (2005) Fine-scale specificity of cortical networks depends on inhibitory cell type and connectivity. Nat Neurosci 8:1552-1559. 